\title{
A Study over the Hyperbolic and Logarithmic Monomolecular, Logistic and Gompertz Growth
} Models

\section{Mehmet KORKMAZ ${ }^{*}$}

ABSTRACT: In this study in addition to classical monomolecular, logistic and Gompertz models, their hyperbolic and logarithmic growth models were found. After that it is searched the effect of these hyperbolic and logarithmic growth models on the choice of appropriate growth model by using two separate data sets. For this purpose, classical monomolecular, logistic and Gompertz growth models and their hyperbolic and logarithmic growth models are compared with some model selection criteria such as coefficient of determination, error sum of squares. For two data sets it is found that the results of these hyperbolic and logarithmic growth models are better than the results of these growth models. Thus, it is considered that these hyperbolic and logarithmic growth models can be used in addition to these growth models. Even the results of these hyperbolic and logarithmic growth models were found the same for both data sets. In addition, some other hyperbolic and logarithmic growth models can be investigated for getting the best model choice.

Keywords: Hyperbolic, Logarithmic, Growth Models, Monomolecular, Logistic, Gompertz

${ }^{1}$ Mehmet KORKMAZ (Orcid ID: 0000-0002-7488-0552), Ordu Üniversitesi, Fen Edebiyat Fakültesi, Matematik Bölümü, Ordu, Türkiye

*Sorumlu Yazar/Corresponding Author: Mehmet KORKMAZ, e-mail: mkorkmaz52@yahoo.com 


\section{INTRODUCTION}

Growth is one of the well-known features in biological creatures (Burkhart and Strub,1974). Growth models describe the changing size of something over time. The process of developing a mathematical model is called mathematical modeling. A model may help to explain a system and to study the effects of different components, and also to make predictions about behavior (Oyamakin and Chukwu, 2015).

A mathematical description of a real world system is often referred to as a mathematical model. A system can be formally defined as a set of elements also called components. A system can be formally defined as a set of elements also called components A set of trees in a forest stand, producers and consumers in an economic system are examples of components. The elements (components) have certain characteristics or attributes and these attributes have numerical or logical values. Among the elements, relationships exist and consequently the elements are interacting. The state of a system is determined by the numerical or logical values of the attributes of the system elements (Oyamakin and Chukwu, 2015). Experimenting on the state of a system with a model over time is termed simulation (Kansal et al., 2000).

In this study, an alternative nonlinear growth models called the hyperbolic and logarithmic monomolecular, Logistic and Gompertz were introduced and compared with the classical monomolecular (Brody,1945), Logistic (Ricker, 1979) and Gompertz models (Winsor, 1932) which are widely used.

\section{Monomolecular Model}

Since the origin of the monomolecular growth model suggested that the growth is directly proportional to the difference of the maximum sustainable constant A and the growth, this growth model starts the following differential equation:

$\frac{\partial \mathrm{y}}{\partial \mathrm{x}}=r(A-y)$

where $\mathrm{y}$ is the value of growth, $\mathrm{x}$ is time, $\mathrm{r}$ is the proportion about growth, $\mathrm{A}$ is maximum sustainable growth

This differential equation produces a model of the following form:

$\mathrm{y}=A\left(1-b e^{-r x}\right)$

where $\mathrm{b}$ is a constant

The monomolecular model in equation 2 is found the following operations:

Separating the variables in equation 1 we get:

$\frac{\mathrm{dy}}{\mathrm{A}-\mathrm{y}}=r d x$

Integrating both sides we get:

$-\ln (A-y)=r x+C_{1}$

$\ln (A-y)^{-1}=r x+C_{1}$

where $C_{1}$ is constant

Taking the exponential of both sides we get:

$(A-y)^{-1}=B e^{r x}$ 
$\frac{1}{A-y}=B e^{r x}$

where $B=e^{C_{1}}$

Multiply both sides by A-y we get:

$1=A B e^{r x}-y B e^{r x}$

$y B e^{r x}=A B e^{r x}-1$

Divide by $\mathrm{B} e^{r x}$ we get monomolecular model as

$y=A\left(1-b e^{-r x}\right)$

where $b=(A B)^{-1}$

\section{Logistic Model}

The origin of the logistic growth model is described by the following equation:

$\frac{\partial \mathrm{y}}{\partial \mathrm{x}}=r y\left(1-\frac{y}{A}\right)$

where $\mathrm{y}$ is the value of growth, $\mathrm{x}$ is time, $\mathrm{r}$ is the proportion about growth, $\mathrm{A}$ is maximum sustainable growth

This differential equation produces the model called logistic model in equation 4 :

$\mathrm{y}=\frac{A}{1+b e^{-r x}}$

where $\mathrm{b}$ is a constant

The logistic model in equation 4 is found the following operations:

Separating the variables in equation 3 we get:

$\frac{\mathrm{Ady}}{\mathrm{y}(\mathrm{A}-\mathrm{y})}=r d x$

Integrating both sides we get:

$\int \frac{\mathrm{Ady}}{\mathrm{y}(\mathrm{A}-\mathrm{y})}=\int r d x$

$\int\left(\frac{1}{y}+\frac{1}{A-y}\right) d y=\int r d x$

$\ln (y)-\ln (A-y)=r x+C_{1}$

$\ln \left(\frac{A-y}{y}\right)=-r x-C_{1}$

where $C_{1}$ is constant

Taking the exponential of both sides we get:

$\frac{A-y}{y}=b e^{-r x}$

$\frac{A}{y}-1=b e^{-r x}$

where $b=e^{-C_{1}}$ 
After this we get:

$\frac{A}{y}=b e^{-r x}+1$

and then get the logistic model as

$y=\frac{A}{1+b e^{-r x}}$

\section{Gompertz Model}

The origin of the Gompertz growth model is described by the following equation:

$\frac{\partial \mathrm{y}}{\partial \mathrm{x}}=\operatorname{ryln}\left(\frac{A}{y}\right)$

where $\mathrm{y}$ is the value of growth, $\mathrm{x}$ is time, $\mathrm{r}$ is the proportion about growth, $\mathrm{A}$ is maximum sustainable growth

This differential equation produces the model called the Gompertz model in equation 6:

$\mathrm{y}=A e^{-b e^{-r x}}$

where $\mathrm{b}$ is a constant

The Gompertz model in equation 6 is found the following operations:

Separating the variables in equation 5 we get:

$\frac{\mathrm{dy}}{\mathrm{y} \ln \left(\frac{A}{y}\right)}=r d x$

Integrating both sides we get:

$\int \frac{\mathrm{dy}}{\mathrm{y} \ln \left(\frac{A}{y}\right)}=\int r d x$

If we say $\mathrm{u}=\ln \left(\frac{A}{y}\right)$ and $d u=\frac{-\frac{A}{y^{2}}}{\frac{A}{y}} d y=\frac{-d y}{y}$, then we can get

$-\int \frac{\mathrm{du}}{\mathrm{u}}=\int r d x$

and

$-\ln u=r x+C_{2}$

Taking the exponential of both sides we get:

$u=b e^{-r x}$

$\ln \left(\frac{A}{y}\right)=b e^{-r x}$

where $b=e^{-C_{2}}$

Again taking the exponential of both sides we get:

$\frac{A}{y}=e^{b e^{-r x}}$

and then get the Gompertz model as

$\mathrm{y}=A e^{-b e^{-r x}}$ 


\section{MATERIALS AND METHODS}

\section{Materials}

Two different data sets were used in this study. The first one is the mean fork length measurements of female and male flathead trout (Salmo platycephalus). The second one is the length of the eucalyptus plant.

In this study, the mean fork length measurements of the flathead trout (Salmo platycephalus), which is listed in the IUCN red list of threatened species, which were sampled from the Zamanti Stream of Seyhan River were used (Kara et al., 2011). For the presentation of the models, the measurements of the fork lengths $(\mathrm{cm})$ in the age-structured female and male of S. platycephalus from Zamanti Stream of the River Seyhan in Table 1 in their articles were used in this study in Table 1.

Table 1. Observed mean fork lengths of S. platycephalus According to the Gender

\begin{tabular}{lcccccccccc}
\hline Sex/Age (year) & $\mathbf{1}$ & $\mathbf{2}$ & $\mathbf{3}$ & $\mathbf{4}$ & $\mathbf{5}$ & $\mathbf{6}$ & $\mathbf{7}$ & $\mathbf{8}$ & $\mathbf{9}$ & $\mathbf{1 0}$ \\
\hline Female & 13.68 & 18.03 & 21.63 & 25.55 & 28.29 & 30.85 & 33.37 & 36.03 & 38.30 & 40.0 \\
Male & 15.15 & 18.72 & 20.91 & 25.11 & 27.97 & 30.88 & 33.13 & 35.94 & 37.04 & 39.20 \\
\hline
\end{tabular}

In this study, the data taken from the tree, E. Camaldulensis Dehn. were used for the growth model in Table 2. The data set was taken from the study of Yildizbakan (2015).

Table 2. The height growth value of the trees (E. Camaldulensis Dehn) according to year

\begin{tabular}{lcccccccccc}
\hline \multicolumn{10}{c}{ The height growth value of the trees (E. Camaldulensis Dehn) } \\
\hline Planting Age (year) & $\mathbf{0}$ & $\mathbf{1}$ & $\mathbf{2}$ & $\mathbf{3}$ & $\mathbf{4}$ & $\mathbf{5}$ & $\mathbf{6}$ & $\mathbf{7}$ & $\mathbf{8}$ & $\mathbf{9}$ \\
\hline Height Growth $(\mathrm{m})$ & 0.41 & 3.23 & 7.45 & 11.41 & 14.83 & 18.11 & 18.95 & 19.69 & 21.50 & 23.40 \\
\hline
\end{tabular}

\section{Methods}

The hyperbolic functions have similar names to the trigonometric functions, but they are defined in terms of the exponential function (Oyamakin and Chukwu, 2015).

Thus the hyperbolic sine function and its inverse provide an alternative method for evaluating:

$\int \frac{1}{\sqrt{1+x^{2}}} d x$

say $x=\sinh (u)$ and then $d x=\cosh (u) d u$

$\int \frac{1}{\sqrt{1+x^{2}}} d x=\operatorname{arcsinh}(x)+C_{3}$

where $C_{3}$ is constant

Also say $\mathrm{x}=\tan \mathrm{u},\left(\frac{-\pi}{2}<u<\frac{\pi}{2}\right) \mathrm{dx}=\left(1+\tan ^{2} \mathrm{u}\right) \mathrm{du}$

$\int \frac{1}{\sqrt{1+x^{2}}} d x=\ln \left|x+\sqrt{1+x^{2}}\right|+C_{4}$

where $C_{4}$ is constant

From equations 8 and 9, we get:

$\operatorname{arcsinh}(x)=\ln \left|x+\sqrt{1+x^{2}}\right|+C_{4}-C_{3}$

for all $x$. Evaluating both sides of this equality at $x=0$, we get

$0=\operatorname{arcsinh}(0)=\ln |1|+C_{3}-C_{2}$ 
So $C_{4}-C_{3}=0$ and

$\operatorname{arcsinh}(x)=\ln \left|x+\sqrt{1+x^{2}}\right|$

for all x. Since the hyperbolic sine function is defined in terms of the exponential function, we should not find it surprising that the inverse hyperbolic sine function may be expressed in terms of the natural logarithm function (Oyamakin and Chukwu, 2015).

\section{Hyperbolic and Logarithmic Monomolecular Growth Model}

The origin of the hyperbolic and logarithmic monomolecular growth models suggested that in addition to constant $r$ in the original monomolecular model and the term

$\frac{d}{\sqrt{1+x^{2}}}$

which is multiplied by the difference of the maximum sustainable constant A and the growth is added where $d$ is proportion about the hyperbolic and logarithmic monomolecular growth, respectively. And so the hyperbolic and logarithmic monomolecular growth models start the following differential equation:

$\frac{\partial \mathrm{y}}{\partial \mathrm{x}}=\left(r+\frac{d}{\sqrt{1+x^{2}}}\right)(A-y)$

This differential equation produces two types of models in equation 13 and equation 14, respectively:

$\mathrm{y}=A\left(1-b e^{-r x-\operatorname{darcsinh}(x)}\right)$

$\mathrm{y}=A\left(1-b e^{-r x}\left(x+\sqrt{1+x^{2}}\right)^{-d}\right)$

The hyperbolic and logarithmic monomolecular models in equation 13 and equation 14 are found the following operations, respectively:

Separating the variables in equation 12 we get:

$\frac{\mathrm{dy}}{\mathrm{A}-\mathrm{y}}=\left(r+\frac{d}{\sqrt{1+x^{2}}}\right) d x$

Integrating both sides we get:

$-\ln (A-y)=r x+d \operatorname{arcsinh}(x)+C_{5}$

$\ln (A-y)^{-1}=r x+d \operatorname{arcsinh}(x)+C_{5}$

and

$-\ln (A-y)=r x+d \ln \left(x+\sqrt{1+x^{2}}\right)+C_{5}$

$\ln (A-y)^{-1}=r x+d \ln \left(x+\sqrt{1+x^{2}}\right)+C_{5}$

where $C_{5}$ is constant

Taking the exponential of both sides of equation 15 and equation 16, respectively we get:

$(A-y)^{-1}=B e^{r x+\operatorname{darcsinh}(x)}$

$\frac{1}{A-y}=B e^{r x+\operatorname{darcsinh}(x)}$

and

$(A-y)^{-1}=B e^{r x+d \ln \left(x+\sqrt{1+x^{2}}\right)}$

$\frac{1}{A-y}=B e^{r x}\left(x+\sqrt{1+x^{2}}\right)^{d}$ 
respectively where $B=e^{C_{5}}$

Multiply both sides of equation 17 and equation 18 by (A-y), respectively we get:

$1=A B e^{r x+\operatorname{darcsinh}(x)}-y B e^{r x+\operatorname{darcsinh}(x)}$

$y B e^{r x+\operatorname{darcsinh}(x)}=A B e^{r x+\operatorname{darcsinh}(x)}-1$

and

$1=A B e^{r x}\left(x+\sqrt{1+x^{2}}\right)^{d}-y B e^{r x}\left(x+\sqrt{1+x^{2}}\right)^{d}$

$y B e^{r x}\left(x+\sqrt{1+x^{2}}\right)^{d}=A B e^{r x}\left(x+\sqrt{1+x^{2}}\right)^{d}-1$

respectively.

Divide equation 19 and equation 20 by $\mathrm{B}^{r x+\operatorname{darcsinh}(x)}$ and $e^{r x}\left(x+\sqrt{1+x^{2}}\right)^{d}$, respectively we get the hyperbolic and logarithmic monomolecular models as

$y=A\left(1-b e^{-r x-\operatorname{darcsinh}(x)}\right)$

and

$\mathrm{y}=A\left(1-b e^{-r x}\left(x+\sqrt{1+x^{2}}\right)^{-d}\right.$

respectively where $\mathrm{b}=(\mathrm{AB})^{-1}$

\section{Hyperbolic and Logarithmic Logistic Growth Model}

The origin of the hyperbolic and logarithmic logistic growth models suggested that in addition to constant $\mathrm{r}$ in the original logistic model and the term

\section{$\frac{d}{\sqrt{1+x^{2}}}$}

which is multiplied by the multiplication of the difference of the maximum sustainable constant $\mathrm{A}$ and the growth and the division of the growth and the maximum sustainable constant $\mathrm{A}$ is added where $\mathrm{d}$ is proportion about hyperbolic and logarithmic logistic growth.

And so the hyperbolic and logarithmic logistic growth models start the following differential equation:

$\frac{\partial \mathrm{y}}{\partial \mathrm{x}}=\left(r+\frac{d}{\sqrt{1+x^{2}}}\right) \frac{y}{A}(A-y)$

This differential equation produces two types of models in equation 22 and equation 23, respectively:

$y=\frac{A}{1+b e^{-r x-\operatorname{darcsinh}(x)}}$

$y=\frac{A}{1+b e^{-r x}\left(x+\sqrt{1+x^{2}}\right)^{-d}}$

The hyperbolic and logarithmic logistic growth models in equation 22 and equation 23 are found the following operations, respectively:

Separating variables in equation 21 we get:

$\frac{\text { Ady }}{\mathrm{y}(\mathrm{A}-\mathrm{y})}=\left(r+\frac{d}{\sqrt{1+x^{2}}}\right) d x$ 
Integrating both sides we get:

$\int \frac{\mathrm{Ady}}{\mathrm{y}(\mathrm{A}-\mathrm{y})}=\int\left(r+\frac{d}{\sqrt{1+x^{2}}}\right) d x$
$\ln (y)-\ln (A-y)=r x+\operatorname{darcsinh}(x)+C_{6}$
$\ln \left(\frac{A-y}{y}\right)=-r x-\operatorname{darcsinh}(x)-C_{6}$

and

$\ln (y)-\ln (A-y)=r x+d \ln \left(x+\sqrt{1+x^{2}}\right)+C_{6}$

$\ln \left(\frac{A-y}{y}\right)=-r x-d \ln \left(x+\sqrt{1+x^{2}}\right)-C_{6}$

where $C_{6}$ is constant

Taking the exponential of both sides of equation 24 and equation 25 , respectively we get:

$\frac{A-y}{y}=b e^{-r x-\operatorname{darcsinh}(x)}$

$\frac{A}{y}-1=b e^{-r x-\operatorname{darcsinh}(x)}$

and

$\frac{A-y}{y}=b e^{-r x-d \ln \left(x+\sqrt{1+x^{2}}\right)}$

$\frac{A}{y}-1=b e^{-r x-d \ln \left(x+\sqrt{1+x^{2}}\right)}$

respectively where $b=e^{-C_{5}}$

After this we get:

$\frac{A}{y}=b e^{-r x-d \operatorname{arcsinh}(x)}+1$

and

$\frac{A}{y}=b e^{-r x}\left(x+\sqrt{1+x^{2}}\right)^{-d}+1$

And then get the hyperbolic and logarithmic logistic growth models as

$y=\frac{A}{1+b e^{-r x-\operatorname{darcsinh}(x)}}$

and

$y=\frac{A}{1+b e^{-r x}\left(x+\sqrt{1+x^{2}}\right)^{-d}}$

respectively.

\section{Hyperbolic and Logarithmic Gompertz Growth Model}

The origin of the hyperbolic and logarithmic Gompertz growth models suggested that in addition to constant $r$ in the original Gompertz model and the term

$\frac{d}{\sqrt{1+x^{2}}}$

which is multiplied by the multiplication of the logarithm of the division of the maximum sustainable constant $\mathrm{A}$ and the growth and the growth is added where $\mathrm{d}$ is proportion about the hyperbolic and logarithmic Gompertz growth. 
And so the hyperbolic and logarithmic Gompertz growth models start the following differential equation:

$\frac{\partial \mathrm{y}}{\partial \mathrm{x}}=\left(r+\frac{d}{\sqrt{1+x^{2}}}\right) y \ln \left(\frac{A}{y}\right)$

This differential equation produces two types of models in equation 29 and equation 30, respectively:

$y=A e^{-b e^{-r x-\operatorname{darcsinh}(x)}}$

and

$y=A e^{-b e^{-r x}\left(x+\sqrt{1+x^{2}}\right)^{-d}}$

The hyperbolic and logarithmic Gompertz growth model in equation 29 and equation 30 are found the following operations, respectively:

Separating the variables in equation 28 we get:

$\frac{\mathrm{dy}}{\mathrm{y} \ln \left(\frac{A}{y}\right)}=\left(r+\frac{d}{\sqrt{1+x^{2}}}\right) d x$

Integrating both sides we get:

$\int \frac{\text { Ady }}{y \ln \left(\frac{A}{y}\right)}=\int\left(r+\frac{d}{\sqrt{1+x^{2}}}\right) d x$

Solving these integrals in equation 31 we get

$\ln \left(\frac{A}{y}\right)=b e^{-r x-\operatorname{darcsinh}(x)}$

and

$\ln \left(\frac{A}{y}\right)=b e^{-r x}\left(x+\sqrt{1+x^{2}}\right)^{-d}$

where $\mathrm{b}$ is constant

Taking the exponential of both sides of equation 32 and equation 33, respectively we get:

$\frac{A}{y}=e^{b e^{-r x-d a r c s i n h}(x)}$

and

$\frac{A}{y}=e^{b e^{-r x\left(x+\sqrt{1+x^{2}}\right)^{-d}}}$

respectively and then get the hyperbolic and logarithmic Gompertz growth models as

$y=A e^{-b e^{-r x-d \operatorname{arcsinh}(x)}}$

and

$y=A e^{-b e^{-r x}\left(x+\sqrt{1+x^{2}}\right)^{-d}}$

respectively.

\section{RESULTS AND DISCUSSION}

Since the hyperbolic and logarithmic growth models in this study are exactly same models, the parameters and the results of known model selection criteria such as error sum of squares (SSE), and coefficient of determination $\left(R^{2}\right)$ of them are exactly same.

By using the Tables 1 and 2 of these two sets of data, in Tables 3 and 5 the parameters of Monomolecular, Logistic and Gompertz growth models and their hyperbolic and logarithmic growth models are calculated. All calculations were made with the maple package program. 
To make a comparison; error sum of squares, and coefficient of determination are used from known model selection criteria. Calculations made are tabulated.

Table 1 shows the mean fork length measurements of female and male flathead trout (Salmo platycephalus). While the model parameters calculated according to these values are given in Table 3 , the error sum of squares and determination coefficients of the models calculated are given in Table 4. Tables 3 and Table 4 are calculated according to the values in Table 1.

Table 3 Calculated the Model Parameters According to the Gender

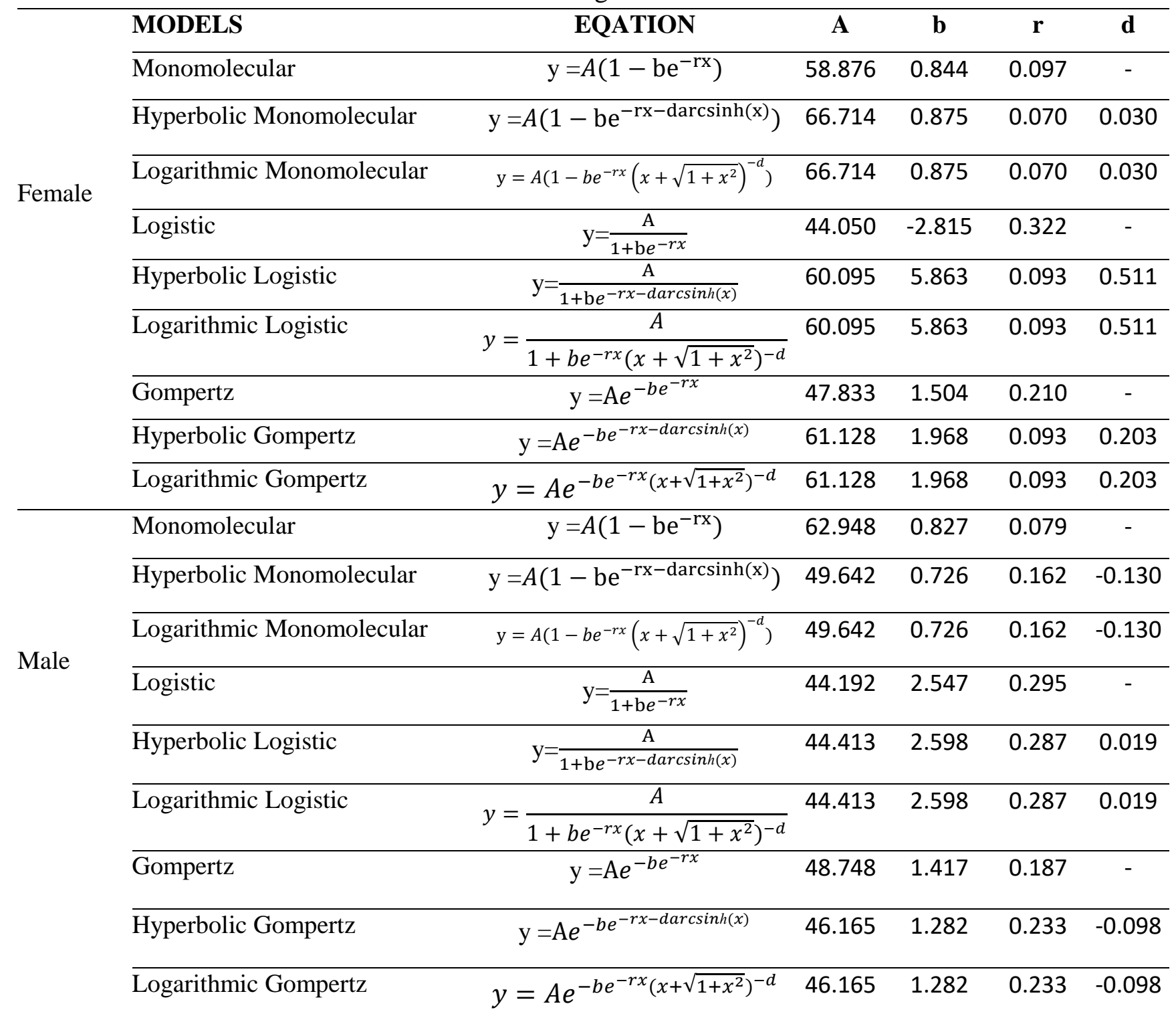

According to Table 4, the hyperbolic and logarithmic models of the models used in this study has approximately the same least error sum of squares and the highest determination coefficient. Therefore, it can be said that the most suitable models for the data set are the hyperbolic and logarithmic ones.

Table 2 shows the growth values of the eucalyptus plant as a whole. While the model parameters calculated according to these values are given in Table 5, the error sum of squares and the determination coefficients of the models calculated are given in Table 6.

Table 5 and Table 6 are calculated according to the values in Table 2. 
Table 4 Error Sum of Squares and Determination Coefficients of the Models Calculated

\begin{tabular}{|c|c|c|c|c|}
\hline \multirow[b]{2}{*}{ MODELS } & \multicolumn{2}{|r|}{ Female } & \multicolumn{2}{|r|}{ Male } \\
\hline & $\begin{array}{c}\text { Error Sum } \\
\text { Squares }\end{array}$ & $\begin{array}{c}\text { Determination Coefficients } \\
\text { of the models }\left(R^{2}\right)\end{array}$ & $\begin{array}{c}\text { Error Sum } \\
\text { Squares }\end{array}$ & $\begin{array}{c}\text { Determination Coefficients } \\
\text { of the models }\left(R^{2}\right)\end{array}$ \\
\hline Monomolecular & 0.368 & 0.9995 & 1.774 & 0.9971 \\
\hline Hyperbolic Monomolecular & 0.303 & 0.9996 & 1.154 & 0.9981 \\
\hline Logarithmic Monomolecular & 0.303 & 0.9996 & 1.154 & 0.9981 \\
\hline Logistic & 2.284 & 0.9967 & 1.089 & 0.9982 \\
\hline Hyperbolic Logistic & 0.301 & 0.9996 & 1.087 & 0.9982 \\
\hline Logarithmic Logistic & 0.301 & 0.9996 & 1.087 & 0.9982 \\
\hline Gompertz & 1.059 & 0.9985 & 1.222 & 0.9980 \\
\hline Hyperbolic Gompertz & 0.305 & 0.9996 & 1.090 & 0.9982 \\
\hline Logarithmic Gompertz & 0.305 & 0.9996 & 1.090 & 0.9982 \\
\hline
\end{tabular}

Table 5 Model Parameters Calculated

\begin{tabular}{lccccc}
\hline MODELS & EQATION & A & b & r & d \\
\hline Monomolecular & $\mathrm{y}=A\left(1-\mathrm{be}^{-\mathrm{rx}}\right)$ & 29.999 & 1.014 & 0.166 & - \\
Hyperbolic Monomolecular & $\mathrm{y}=A\left(1-\mathrm{be}^{-\mathrm{rx}-\mathrm{darcsinh}(\mathrm{x})}\right)$ & 24.609 & 0.986 & 0.357 & -0.253 \\
Logarithmic Monomolecular & $\mathrm{y}=A\left(1-b e^{-r x}\left(x+\sqrt{1+x^{2}}\right)^{-d}\right)$ & 24.609 & 0.986 & 0.357 & -0.253 \\
Logistic & $\mathrm{y}=\frac{\mathrm{A}}{1+\mathrm{b} e^{-r x}}$ & 21.887 & 10.685 & 0.781 & - \\
Hyperbolic Logistic & $\mathrm{y}=\frac{\mathrm{A}}{1+\mathrm{b} e^{-r x-\operatorname{darcsinh}(x)}}$ & 29.104 & 39.955 & -0.061 & 1.894 \\
Logarithmic Logistic & $y=\frac{A}{1+b e^{-r x}\left(x+\sqrt{1+x^{2}}\right)^{-d}}$ & 29.104 & 39.955 & -0.061 & 1.894 \\
Gompertz & $\mathrm{y}=\mathrm{A} e^{-b e^{-r x}}$ & 23.071 & 3.118 & 0.488 & - \\
Hyperbolic Gompertz & $\mathrm{y}=\mathrm{A} e^{-b e^{-r x-\operatorname{darcsinh}(x)}}$ & 30.054 & 4.926 & 0.062 & 0.804 \\
Logarithmic Gompertz & $y=A e^{-b e^{-r x}\left(x+\sqrt{1+x^{2}}\right)^{-d}}$ & 30.054 & 4.926 & 0.062 & 0.804 \\
\hline
\end{tabular}

It is seen in Table 6 that the model with the lowest sum of error squares and the highest determination coefficient is the hyperbolic Gompertz growth model.

Table 6 Error Sum of Squares and Determination Coefficients of the Models Calculated

\begin{tabular}{lcc}
\hline MODELS & Error Sum Squares & Determination Coefficients of the models $\left(\boldsymbol{R}^{\mathbf{2}}\right)$ \\
\hline Monomolecular & 5.266 & 0.9908 \\
Hyperbolic Monomolecular & 2.608 & 0.9954 \\
Logarithmic Monomolecular & 2.608 & 0.9954 \\
Logistic & 8.713 & 0.9847 \\
Hyperbolic Logistic & 2.417 & 0.9958 \\
Logarithmic Logistic & 2.417 & 0.9958 \\
Gompertz & 3.917 & 0.9931 \\
Hyperbolic Gompertz & 2.301 & 0.9960 \\
Logarithmic Gompertz & 2.301 & 0.9960 \\
\hline
\end{tabular}




\section{CONCLUSION}

In this study, three different sigmoidal growth models, which are frequently found in scientific studies, and their hyperbolic growth models have been used. These models have been applied to two different data sets. It has been determined which model is suitable according to the known model selection criteria. It is shown that the results are compatible with each other. It is shown that the appropriate models for both sets of data are their hyperbolic and Logarithmic ones according to the model selection criteria used in this study. That's instead of the growth models used in this study, the use of their hyperbolic and Logarithmic growth models provided the least error sum of squares and the maximal determination coefficients for two data sets. In other words, hyperbolic and Logarithmic growth models have the lowest sum of error squares and the highest determination coefficient according to their classical growth models. In addition, the lowest sum of error squares and the highest determination coefficient of hyperbolic and Logarithmic growth models are the same. Therefore, the researchers can use their hyperbolic and Logarithmic growth models in addition to the growth models for getting better results. In this study, instead of comparing the models with each other, hyperbolic and logarithmic models were compared with the same model. In addition, for the other growth models not used in this study, their hyperbolic and logarithmic growth models can be investigated for getting the best model choice.

\section{REFERENCES}

Brody S, 1945. Bioenergetics and Growth, Rheinhold Publishing, New York.

Burkhart HE, Strub MR, 1974. A model for simulation or planted loblolly pine stands. In growth models for tree and stand simulation. Edited by J. Fries. Royal College of Forestry, Stockholm, Sweden. Pp.128135. Model of forest growth. J. Ecol.60:849-873.

Kansal AR, Torquato S, Harsh GR, 2000. Simulated brain tumor growth dynamics using a three dimentional cellular automaton. J Theor Biol, 203:367-382.

Kara C, Alp A, Can F, 2011. Growth and Reproductive Properties of Flathead Trout (Salmo platycephalus Bhenke, 1968) Population from Zamanti Stream, Seyhan River, Turkey. Turkish Journal of Fisheries and Aquatic Sciences, 11: 367-375.

Oyamakin, SO, Chukwu AU, 2015. On the Hyperbolic Monomolecular Growth Model in Height/Diameter Growth of PINES, Journal of Applied Mathematics, Statistics and Informatics, 11(1):5-17.

Ricker, WE, 1979. Growth rates and models, Fish Physiol, 8: 677-743.

Winsor, CP, 1932. The Gompertz curve as a growth curve, Proc. Natl. Acad. Sci.,18(1): 1-8.

Yildizbakan A, 2005. Analysis on mathematical models of tree growth and comparison of these models, MSc Thesis, Institute of Natural and Applied Sciences, University of Cukurova, Turkey, (in Turkish, with abstract in English). 